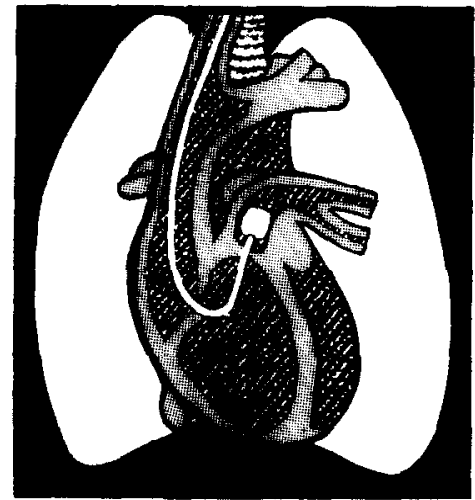

\title{
CASE 5-1991 \\ A 44-Year-Old Woman Was Admitted for Elective Replacement of the Mitral and Aortic Valves. Could There Be Another Problem?
}

\author{
Robert A. Stein, Jr, MD, Steven N. Landau, MD, Roger S. Wilson, MD, \\ and John C. Wain, MD
}

\section{Case Presentation}

A 44-year-old woman was admitted for elective repair/ replacement of her mitral and aortic valves. She had developed rheumatic fever as a child, and since the age of 18 a cardiac murmur had been noted. At approximately age 36 , the patient recalled the onset of dyspnea with exertion. The symptoms progressed in intensity despite medical management, and her lifestyle activities were curtailed accordingly.

Eighteen months prior to admission, the patient underwent cardiac catheterization at another hospital. Significant mitral stenosis (valve area, $1 \mathrm{~cm}^{2}$ ) and noncontributory mild aortic insufficiency were identified. Percutaneous mitral valvuloplasty increased the valve area, but with incomplete resolution of the symptoms. Over the next year, the patient incurred several episodes of atrial fibrillation with a rapid ventricular response. Digoxin and furosemide were prescribed with maintenance of sinus rhythm. However, the dyspnea and exercise intolerance progressed. Three months prior to admission, a second percutaneous mitral valvuloplasty was performed. The patient described minimal symptomatic relief. Referral to this institution was initiated for evaluation and surgical intervention.

At admission, the patient's chief complaint was marked dyspnea associated with minimal exertion. Except for a 1-year history of cough productive of scant white sputum, she denied all other constitutional symptoms, including fever, hemoptysis, hoarseness, stridor, wheezing, orthopnea, and paroxysmal nocturnal dyspnea. Medications at admission included digoxin, furosemide, lisinopril, and potassium. She had had no previous general anesthetics or surgical procedures and there was no history of trauma. The patient had lived in central Africa for several years, working as a missionary. She did not use tobacco or alcohol.

Robert A. Stein, Jr, MD, Steven N. Landau, MD, Department of Anesthesiology, University of Michigan Medical Center, Ann Arbor, MI; Roger S. Wilson, MD, Department of Anesthesia, Harvard Medical School and Massachusetts General Hospital, Boston, MA; John C. Wain, MD, Department of Thoracic Surgery, Massachusetts General Hospital, Boston, MA.

Address reprint requests to Robert A. Stein, Jr, MD, Department of Anesthesiology, University of Michigun Medical Center, 1500 E Medical Center Dr, Ann Arbor, MI 48109-0048.

Copyright 1991 by W.B. Saunders Company 1053-0770/91/0505-0019\$03.00/0
The family history was not contributory for anesthetic abnormalities.

The examination showed a healthy-appearing white woman in no acute distress. The vital signs included normal values for heart rate ( 80 beats $/ \mathrm{min}$ and regular), blood pressure $(110 / 70 \mathrm{~mm} \mathrm{Hg})$, respirations (14 breaths/min and unlabored), and temperature $\left(37.5^{\circ} \mathrm{C}\right)$. She weighed 70 $\mathrm{kg}$ and was 65 in tall. The head and neck examination showed full excursion of the mandible with clear visualization of the uvula and tonsillar pillars. Full flexion and extension of the cervical spine were noted. There were no palpable neck masses. The cardiac examination showed a grade III/VI systolic ejection murmur at the right base and a grade II/VI middiastolic murmur at the apex. No evidence of right or left heart failure existed. The lungs were clear to auscultation and the remainder of the physical examination was within normal limits.

Pertinent laboratory data included normal serum electrolytes, blood urea nitrogen, and creatinine. The hematocrit was $38 \%$ and the platelet count $262,000 / \mu \mathrm{L}$. The electrocardiogram showed a sinus rhythm at 68 beats $/ \mathrm{min}$, axis $+60^{\circ}$. and left atrial enlargement. The chest radiograph was interpreted as normal. Pertinent cardiac catheterization data, obtained 3 months prior to admission, included findings of "moderate-severe mitral stenosis and mild aortic insufficiency." Contributing echocardiographic data included a thickened mitral valve (area, $1.5 \mathrm{~cm}^{2}$ ) with regurgitation and a thickened aortic valve with insufficiency. Left ventricular function was normal and left atrial enlargement (diameter, $55 \mathrm{~mm}$ ) was described.

On the morning of surgery, the patient was given morphine sulphate, $8 \mathrm{mg}$ intramuscularly (IM), and lorazepam, $2 \mathrm{mg}$, orally. After routine monitors were placed prior to the induction of anesthesia, a left radial artery catheter and a flow-directed pulmonary artery catheter were inserted. The systemic blood pressure ranged between 110/70 and $130 / 85 \mathrm{~mm} \mathrm{Hg}$. The pulmonary artery pressure was $38 / 15$ $\mathrm{mm} \mathrm{Hg}$, the pulmonary capillary wedge pressure was $15 \mathrm{~mm}$ $\mathrm{Hg}$, and a thermodilution cardiac output was $5.0 \mathrm{~L} / \mathrm{min}$.

Following administration of fentanyl, 3,200 $\mu \mathrm{g}$, and vecuronium, $10 \mathrm{mg}$, the lungs were easily ventilated via mask. Although the vocal folds were clearly visualized. serial intubation attempts with size $8-\mathrm{mm}, 7-\mathrm{mm}$, and 6-mm internal diameter endotracheal tubes were unsuccessful, secondary to resistance distal to the vocal folds. Placement of the laryngoscope blade beyond the glottic opening 
illuminated a white circumferential intralumenal subglottic mass. The otolaryngologist was consulted and direct laryngoscopy, bronchoscopy, and esophagoscopy were performed. The findings confirmed the presence of the isolated tracheal mass with an orifice diameter of $6 \mathrm{~mm}$. A tracheostomy distal to this mass was performed while the mask airway was maintained and, after placement of a tracheal tube, the mass was biopsied and cultured. The patient was transferred to the intensive care unit and subsequently separated from mechanical ventilation. She was able to ambulate within another day and, while breathing via the trachcostomy, notcd almost complctc lack of the previously elicited dyspnea with exertion.

The biopsy specimens showed only scar and granulation tissue. No evidence of neoplasm or granulomatous disease was found. All fungal and bacterial studies were negative for pathogens. A computed tomography scan of the neck showed only the proximal intralumenal tracheal mass; no other abnormality was noted. One week later the patient returned to the operating room for tracheal resection. At operative tracheotomy a concentric subglottic stricture involving the first and second tracheal rings was isolated. The mass extended from a point $2 \mathrm{~cm}$ inferior to the vocal folds caudally for $1.5 \mathrm{~cm}$. This tracheal segment was resected and a primary reanastomosis was performed. Prior to closure of the trachea, a nasotracheal tube was passed for postoperative airway management. The patient was extubated awake the next morning without complication and released from the hospital several days later. At the time of discharge, the diagnosis was listed as probable congenital tracheal stenosis with postinfectious exacerbation, etiology unknown.

During the most recent follow-up 3 months postoperatively, no complaints were elicited and unlimited activity was reported. Examination showed normal vocal fold mobility, a 3-mm mound of subglottic scar, and an otherwise normal subglottic airway. Currently, manifestations of the mitral and aortic valvular disease are well controlled with oral medications and no immediate plans for operative repair are under consideration.

\section{DISCUSSION}

\section{Rheumatic Valvular Heart Disease and an Isolated Tracheal Mass*}

Mitral stenosis, when associated with a history of rheumatic fever, may remain clinically silent for up to 20 years following the group A Streptococcal insult.' As the mitral valve orifice decreases in size, progressive symptoms of cardiopulmonary decompensation will become apparent. Increased left atrial pressure, pulmonary vascular engorgement, and pulmonary hypertension are the causative factors associated with the onset of exertional dyspnea, orthopnea, and paroxysmal nocturnal dyspnea. Left atrial enlargement will predispose to the development of atrial fibrillation. In some instances, medical management and percutaneous transluminal balloon valvuloplasty may delay

*Robert A. Stein, Jr, MD, and Steven N. Landau, MD the need for a surgical procedure. Ultimately, valve replacement is warranted when intolerable chamber and/or vascular consequences approach. ${ }^{1,2}$

This patient with severe cardiac catheterization-diagnosed mitral stenosis and mild aortic insufficiency arising secondary to rheumatic fever appeared to develop progressive symptoms of cardiopulmonary decompensation necessitating surgical intervention despite successful mitral valvuloplasty and optimal medical therapy. However, the unrecognized tracheal lesion accounted for much of the acute symptomatology.

Isolated, nonmetastatic, nonextrinsic trachcal masses are uncommon and may arise from congenital, infectious, neoplastic, and traumatic processes. Table 1 contains an overview of these varied entities. ${ }^{3.7}$ Of note, the most common cause of benign tracheal stricture is acquired scarring secondary to postintubation mucosal ulceration. This is usually attributed to excessive tracheal tube cuff pressures and/or prolonged intubation. There is no evidence to suggest a correlation between the history of rheumatic fever and this intratracheal process.

Evaluation of an intratracheal mass should include tracheal tomograms, computed tomography, pulmonary function testing with flow-volume loop studies, bronchoscopy, and arterial blood gas analysis. ${ }^{8,9} \mathrm{~A}$ most important matter regarding the care of a patient with such pathology is a suspicion of its presence. This would be predicated on eliciting a history of bronchospasm, hemoptysis, hoarseness, or stridor. In most cases, the airway must be narrowed to an oritice size of 5 to $6 \mathrm{~mm}$ before signs and symptoms become evident. ${ }^{10}$ Retrospectively, this patient had had a

Table 1. Intratracheal Masses

\begin{tabular}{l} 
Congenital \\
Deformity of tracheal cartilage \\
Fibrous web \\
"Hourglass" stenosis (associated with tracheoesophageal fistula \\
or esophageal atresia) \\
Infectious \\
Diphtheria (Corynebacterium diphtheriae) \\
Rhinoscleroma (Klebsiella rhinoscleromatis) \\
Syphilis (Treponema pallidum) \\
Tuberculosis (Mycobacterium tuberculosis) \\
Typhoid fever (Sa/mone/la typhi) \\
Neoplastic, benign \\
Adenoma \\
Chondroma \\
Fibroma (including neurofibroma) \\
Hamartoma \\
Hemangioma \\
Lipoma \\
Squamous papillorna \\
Tracheopathic osteoplastica (often multicentric) \\
Neoplastic, malignant \\
Adenocarcinoma \\
Cylindroma (adenoid cystic carcinoma) \\
Squamous cell carcinoma \\
Traumatic \\
Scarring/stenosis (endotracheal intubation; sharp/blunt trauma \\
Tracheomalacia \\
\hline
\end{tabular}


productive cough and dyspnea. Thesc symptoms did not specifically identify the culprit lesion and, in fact, were attributed to the mitral valve disease

In the final analysis, a patient with significant rheumatic valvular disease was also troubled with symptoms arising from unsuspected subglottic airway obstruction. Ultimately, the patient achieved an excellent clinical outcome albeit via means different from those originally planned.

\section{COMMENTARY}

\section{Unsuspected Airway Obstruction: A Dilemma for the Anesthesiologist $\dagger$}

This case demonstrates the subtle nature of presentation of airway pathology. Common clinical symptoms, including dyspnea, especially with effort, wheezing, stridor, cough, and difficulty in clearing secretions, although simple and nonspecific, are frequently misdiagnosed. As previously noted, symptoms may be ascribed to other etiologies such as pulmonary pathology, often "asthma," or presumed cardiac disease. In many cases, suspicion that the primary diagnosis is in error arises only when there is failure in response to standard therapies and an index of suspicion of airway pathology.

Physical examination during the preoperative anesthetic visit may not alert the physician to the presence of airway pathology. Audible stridor at rest is generally not present until the airway diameter is markedly reduced. Stridor with a provoked maximal expiratory effort is generally elicited with lesions limiting cross-sectional diameter to the range of 5 to $6 \mathrm{~mm}$. Chest auscultation frequently will show diffuse inspiratory and expiratory wheezing, which is generally similar to that anticipated with typical bronchospasm. As described in this case, auscultation of the lung fields can be entirely normal. Auscultation of the upper cervical airway, especially when the patient augmented flow, may have demonstrated high-pitched inspiratory and cxpiratory sounds characteristic of airway obstruction.

Routine chest $x$-ray will often fail to show pathology, especially with subglottic lesions. Although more specific radiographs such as anteroposterior and lateral cervical views and tomograms might have delineated the extent of pathology, there were no preoperative indications to obtain such studies.

The presence of dyspnea and exercise limitation, although easily attributable to cardiac dysfunction, should always raise the potential question about a pulmonary cause. A simple outpatient screening study such as a flow-volume loop, in this setting of a fixed extrathoracic lesion, would likely show limitation of peak inspiratory and expiratory flow. In addition, it would show the characteristic "plateau" configuration of the inspiratory and expiratory limbs of the flow volume loop. Arterial blood gas analysis is not likely to be helpful at this degree of airway compromise.

Although rare, the inability to pass a "standard-sized" endotracheal tube into what has been presumed to be a normal airway does occur. After several failed attempts with subsequently smaller diameter tubes, the anesthesiolo. gist must decide on the best approach for overall manage ment. The major decision rests among (1) waking tho patient, (2) intubating with the smallest diameter cuffed or uncuffed tube that will comfortably fit into the abnormal airway, and (3) needing a definitive diagnosis. Termination of anesthesia to awaken the patient is obviously governed by the need to proceed with the surgical procedure and the anesthetic technique used during induction. Perhaps the most important element in the decision-making process centers on the need to make a definitive diagnosis about the airway pathology'

Invasive emergency procedures such as cricothyrotomy. "emergency" tracheostomy, and blind transtracheal cannulation are potentially hazardous when the degree and nature of the airway pathology are not known. The most logical and efficient approach, provided that ventilation is adequate, is to proceed with careful visual examination of the airway. This would include direct laryngoscopy and either rigid or fiberoptic bronchoscopy. After bronchoscopy, it is possible to make a decision about management of the airway and whether to treat the pathology immediately in conjunction with the intended surgical procedure or to delay management to a later date after either tracheal dilatation and/or some temporizing surgical procedure have been undertaken.

Anesthetic management for the subsequent, delayed repair must consider not only the airway pathology but the other preexisting medical problems. In general, surgical resection and reconstruction of the upper airway with lesions in the area of the first and second tracheal ring can be expected to be accomplished without major impairment to cardiopulmonary function. Selection of specific monitoring and anesthetic induction/maintenance techniques is governed by the extent of cardiac impairment. A common approach to the induction of anesthesia in a patient with a tracheostomy in place and maintenance of anesthesia with inhalation agent/oxygen would be expected to provide satisfactory anesthesia for the anticipated surgical procedure. In the absence of major cardiopulmonary dysfunction in the postoperative period and with appropriate intraoperative anesthetic management, extubation in the operating room at the termination of the procedure should be considered. Postoperative intubation following resection and reconstruction of the trachea may produce laryngeal edema and trauma at the anastomosis, which, in itself, could create need for reintubation following delayed extubation.

\section{Upper Airway Obstruction: A Surgical Management Plan}

A stenotic lesion in the airway is perhaps the most frequently overlooked etiology of exertional dyspnea. A high index of suspicion, particularly in patients with a "normal chest radiograph," is crucial to improving diagnostic accuracy in these cases.

Most commonly, a prior history of endotracheal intuba-

$\doteqdot$ Roger S. Wilson, MD

ђJohn C. Wain, MD 
tion, trauma, or necrotizing infection (eg, diphtheria) in the laryngotracheal region can be elicited from these patients.) Patients may have a negativc history for any of these etiologic factors, as in the case described here. Patients without a prior history include those with idiopathic laryngotracheal stenoses as well as those with congenital stenoses and airway inflammatory processes such as Wegener's granulomatosis or sarcoidosis. Occasionally, patients with the latter diseases may have other associated otolaryngologic manifestations.

Stridor is indicative of a narrowing in the extrathoracic (or extraparenchymal) airways. However, stridor is generally noted at rest only when the cross-sectional diameter of the airway narrows to less than $6 \mathrm{~mm}$ or when the airway narrowing is asymmetric. In patients with chronic airway stenoses, the pattern of ventilation may be unconsciously altered, so that the inspiratory phase is slow and exaggerated. This minimizes turbulence at the site of narrowing and masks the presence of any associated stridor. However, inspiratory stridor becomes readily apparent in such patients with any exertion and/or increased minute ventilation.

In all instances, the cvaluation of cxertional dyspnca should include a thorough physical examination of both the pulmonary and cardiovascular systems. In particular, auscultation during rapid inspiration and expiration should be performed over the cervical trachea and along the medial border of the right scapula, in addition to the standard lung fields. Inspection of the chest radiograph in such patients should always include a careful review of the visible tracheal air column, from the cervical region to the main bronchi. The intramediastinal portion of the trachea and carina is often best visualized by elevating the film away from the view box at a $30^{\circ}$ angle.

The management of patients with an undiagnosed airway stenosis at the time of anesthetic induction relates in large part to the location of the lesion and the expertise of available personnel. The first step is accurate identification of the process and its location in the trachea. Direct laryngoscopy with a straight-blade laryngoscope should allow full visualization of the transglottic airway. Inspection of the subglottic and more distal airway is performed either directly using the laryngoscopic exposure or with a rigid bronchoscope or telescope passed through the glottis into the trachea. In general, noninflammatory lesions in the subglottic region or proximal trachea, including both neoplasms and extrinsic compression, can be successfully dilated either with a reduced diameter endotracheal tube or the rigid bronchoscope. More fixed inflammatory lesions in the subglottic region require either initial control of the airway distal to the region via cervical tracheostomy or serial dilatation using rigid bronchoscopes, if a skilled endoscopist is present. Cricothyrotomy in such cases is absolutely contraindicated because of the presence of the underlying pathology in the subglottic region. Inflammatory lesions within the upper trachea, distal to the subglottic region, which cannot be dilated, are best managed by cervical tracheostomy performed through, or in very close approximation to the area of stenosis. This is very important in order to preserve uninvolved airway for subsequent reconstructive maneuvers. For more distal tracheal or carinal lesions, rigid bronchoscopy or endotracheal intubation with an uncut standard endotracheal tube for flexible bronchoscopy is necessary to accurately identify the location and type of lesion. In some instances, jet ventilation through distal stenoses is possible with bronchoscopically placed catheters. However, in most cases, dilatation via the rigid bronchoscope and placement of an endotracheal tube beyond the lesion are the most appropriate approaches. Extubation should be performed only when the patient has fully regained spontaneous respirations and the airway distal to the stenosis has been definitively controlled.

Subsequent management of these lesions depends on their etiology. Primary resection and reconstruction is the method of choice in most instances of localized stenoses, either inflammatory or neoplastic. Permanent endotracheal stenting, such as with a silastic tracheal T-tube, is used for lesions that exceed the limits of possible surgical reconstruction. Extrinsic compression is best managed by first relieving the compression and then dealing with the airway lesion independently. In many cases, airway integrity is maintained and no further therapy is needed, whereas in others stenting or resection because of cartilaginous incompetence is required.

\section{REFERENCES}

1. Rahimtonla SH, Pantley GA, Morton MJ: Valvular heart disease, in Stein JH (ed): Internal Medicine. Boston, MA, Little, Brown, 1983, pp 547-571

2. Baim DS: Interventional catheterization techniques, in Braunwald E (ed): Heart Disease. Philadelphia, PA, Saunders, 1988, pp 1979-1994

3. Sardana DS: Congenital tracheal stenosis. Laryngoscope 76:1615-1622, 1966

4. Grillo HC: Obstructive lesions of the trachea. Ann Otol 82:770-771, 1973

5. Norris CM: Tumors of the trachea, bronchi, and esophagus, in Paparella MM, Schumrick DA (eds): Otolaryngology. Philadelphia, PA, Saunders, 1973, pp 740-752
6. Holinger $\mathrm{PH}$, Johnston $\mathrm{KC}$, Basinger CE: Benign stenosis of the trachea. Ann Otol 59:837-859, 1950

7. Dodge TL, Mahaffey JE, Thomas JD: The anesthetic management of a patient with an obstructing intratracheal mass: A case report. Anesth Analg 56:295-298, 1977

8. Benumof JL, Alfery DD: Anesthesia for thoracic surgery, in Miller RD (ed): Anesthesia (ed 2). New York, NY, Churchill Livingstone, 1986, pp 1371-1462

9. Miller RD, Hyatt RE: Obstructing lesions of the larynx and trachea: Clinical and physiological characteristics. Mayo Clin Proc 44:145-161, 1969

10. Benumof JL: Anesthesia for Thoracic Surgery. Philadelphia, PA, Saunders, 1987, p 349 